\title{
Performance evaluation of different mechanisms of production activity control in the context of Industry 4.0
}

\author{
Daniela Costa $^{1}$, Mariana Martins ${ }^{1}$, Susana Martins ${ }^{1}$, Eduarda Teixeira ${ }^{1}$, Andreia \\ Bastos $^{1}$, Ana Rita Cunha ${ }^{1}$, Leonilde Varela1 [0000-0002-2299-1859], José Machado ${ }^{20000-0002-}$ \\ 4917-2474] \\ ${ }^{1}$ University of Minho, Department of Production and Systems, Algoritmi Research Centre \\ ${ }^{2}$ University of Minho, Mechanical Engineering Department, MEtRICs Research Centre \\ leonilde@dps.uminho.pt, jmachado@dem.uminho.pt
}

\begin{abstract}
Production Activity Control (PAC) is fundamental to Production Management, since it allows for meeting deadlines, ensuring product quality and reducing production costs. For these reasons, it is essential for the improvement of enterprise performance to understand the production system and its integrated parts. Another production concept linked to the efficiency of enterprise performance is Industry 4.0. This is the most recent revolution of industry and one of its main goals are related with the integration of production activity control by using information technologies. The objective of this project is to implement three different mechanisms of Production Activity Control in a Flexible Flow Shop (FFS), composed of three stages with three parallel machines each. The mechanisms implemented are Workload Control (WLC), Generic Kanban System (GKS) and Drum-Buffer-Rope (DBR), and all are associated with a make-to-order (MTO) production. Additionally, three independent machine selection criteria are evaluated: Random, Load Hours and Load Units. Simio software is used for the simulation of the production system and results are given by diverse Key Performance Indicators (KPIs). After completing simulations, it can be concluded that DBR is the mechanism of PAC with the best performance for the studied scenarios. However, the scenario with the smallest value of load norm is compromising the performance of WLC. Otherwise, this mechanism would be the one with the best performance. Regarding the machine selection criteria, Load Hours is the criterion with the best performance for almost all the KPIs.
\end{abstract}

Keywords: Production activity control, Industry 4.0, Workload Control, Generic Kanban System, Drum-Buffer-Rope, Simulation, Key Performance Indicators. 


\section{Introduction}

\subsection{Problem Description}

This project consists of the implementation and evaluation of three production activity control mechanisms (Workload Control, Generic Kanban System and Drum-BufferRope) associated to a make-to-order production strategy. The production system is composed by a flexible flow shop where three different products (Product A, Product $\mathrm{B}$ and Product $\mathrm{C}$ ) are processed at three production stages $(\mathrm{i}=1,2$, and 3$)$. On each stage, there are three different parallel machines (Mi1, Mi2 and Mi3) that execute the same operation for each type of product. The production system configuration is shown in Figure 1.

Fig. 1. Production system configuration.

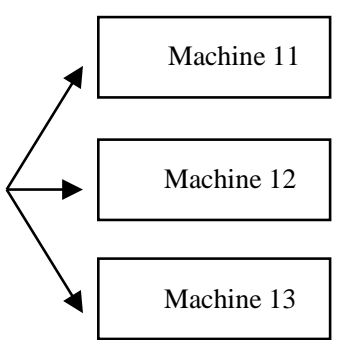

Production

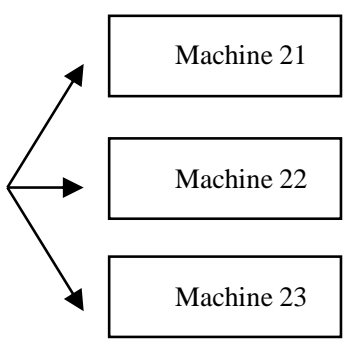

Production

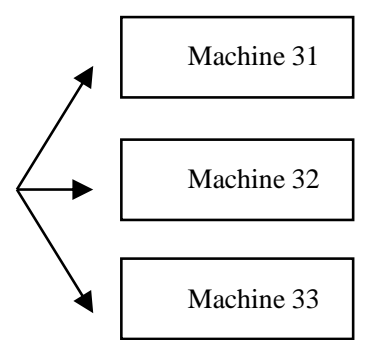

Production

Regarding the products, their proportion in the system is the same (1/3 of each one) and their processing time (hours) is represented in Table 1.

Table 1. Processing time of different products in the system

\begin{tabular}{cccc}
\hline $\begin{array}{l}\text { Product } \\
\text { Type }\end{array}$ & $\begin{array}{c}\text { Machines } \\
\mathbf{M}_{11}, \mathbf{M}_{12}, \\
\mathbf{M}_{13}\end{array}$ & $\begin{array}{c}\text { Machines } \\
\mathbf{M}_{11}, \mathbf{M}_{12}, \\
\mathbf{M}_{13}\end{array}$ & $\begin{array}{c}\text { Machines } \\
\mathbf{M}_{11}, \mathbf{M}_{12}, \\
\mathbf{M}_{13}\end{array}$ \\
\hline Product A & 1 & 1 & 1 \\
Product B & 0,5 & 0,5 & 0,5 \\
Product C & 1,5 & 1,5 & 1,5 \\
\hline
\end{tabular}

The machines' selection can be Random (the selection is random), as a function of Load Hours (the selection is made according to the machines with the lowest workload) or as a function of Load Units (the selection is made depending on the quantity processed by each machine). To evaluate the performance of the different mechanisms, it is necessary to implement them on each different scenario of selection criteria, using the software Simio. For the modelling problem, the characteristics shown in Table 2 are required. 
Table 2. Characteristics of the simulation model

\begin{tabular}{cc}
\hline Inter-arrival Time & EXPO $(0,37) \mathrm{hrs}$ \\
\hline Due Date & TNOW + Unif $(30,60) \mathrm{hrs}$ \\
Processing Time & Truncated 2-Erlang; average (Table 1), \\
Ranking Rule & First Comerage \\
Uptime Between Failures (MTBF) & EXPO (1000) hrs \\
Time to Repair (MTTR) & EXPO (3) hrs \\
Sequencing Rule at the Pool & Planned Release Date (PRD) \\
\hline
\end{tabular}

A dispatching rule (or ranking rule) defines the order in which jobs waiting in the queue of a machine are processed, as soon as the machine becomes unoccupied. In this project, the dispatching rule used is First-In-First-Out (FIFO), which dispatches jobs according to their arrival order at the queue machine [6].

Furthermore, in the pre-shop pool, it is essential to define the order in which jobs will be released into the system. One of the most important sequencing rules is the Planned Release Date (PRD), in which jobs are ordered according to the earliest planned release date, given by the difference between the job due date and the sum of lead times [7].

\subsection{Objectives}

The main goal of this project is to compare the different Production Activity Control mechanisms to discover which performs the best in the system. Additionally, it is pretended to identify which mechanism provides the best results among the diverse selection criteria, as well as which selection criterion is the best performing, for different condition of cards and loads.

This evaluation and comparison will be made through KPIs also implemented in the developed simulation model.

\subsection{Methodology}

In an early phase, the software Simio is used to model the production system, implement the different machine selection criteria and apply the different mechanisms for each criterion.

In a posterior phase, the models created are tested to extract the intended results, to compare the three mechanisms using different selection criteria and vice-versa. 


\section{Key concepts overview}

\section{$2.1 \quad$ Industry 4.0}

According to the Consortium II, Industry 4.0 is "the integration of complex and physical machinery and devices with networked sensors and software, used to predict, control and plan for better business and societal outcomes" [9].

Therefore, Industry 4.0 has been transforming production systems. This concept is composed by several components and the key ones are Cyber-Physical Systems (CPS), Internet of Things (IoT), Internet of Services (IoS) and Smart Factory [12]. Information Technologies allows for a better management and control of production activity, namely, anticipate failures in the system, adapt the production to new scenarios and integrate all activities of the production processes. Thus, factories become smarter, flexible, efficient, dynamic and autonomous, facilitating interconnection among supply chain.

This recent revolution enables the vertical and horizontal integration, since Industry 4.0 supports decision making in a short, medium and long term, creating crosslinking in the different hierarchical levels, as simultaneously provides tools to operational decisions and the intelligent cross-linking within the company and with its partners [15].

Through this concept, a new one emerged, Collaborative Manufacturing (CM) referring to the collaboration among workers from different departments and knowledge fields. This is useful when there is a crucial decision to be made and needs various perspectives.

This paper portrays a situation where Industry 4.0 concepts can have impact in decision making, through simulation tools. This case illustrates how simulation can reproduce a real situation where three mechanisms are evaluated without being implemented in a production system.

\subsection{Production activity control}

Production Activity Control (PAC) comprises a set of rules that plans, guides and controls the production system and has the purpose of effectively using the different production resources [2]. It also considers the fulfilment of demand, deadlines and production programs of a company.

As a short-term function, PAC includes the following phases [3]:

- Job releasing to the production system;

- Load distribution through work centres;

- Job sequencing through work centres;

- Job supervision and control.

Furthermore, it is relevant to distinguish the diverse production systems' classification: Push, Pull or Hybrid. In a Push System, production only starts when the necessary materials to satisfy a production program are available to be processed. Consequently, whenever a job is concluded, it is pushed to the following work centre until all operations are finished. Contrarily, in the Pull System, jobs in the upstream pro- 
duction system phases are pulled by the downstream ones. Therefore, jobs are produced to fulfil demand. Lastly, the Hybrid System is a mix of both.

In this project, the system implemented is the Pull System [5].

\subsection{Production activity control approaches}

According to Stevenson, Hendry, \& Kingsman (2005), there are five different approaches to the PAC [14]:

- Material Requirements Planning (MRP);

- Workload Control (WLC);

- Just in Time (JIT) / Lean Manufacturing;

- $\quad$ Theory of Constraints (TOC);

- $\quad$ Quick Response Manufacturing (QRM).

Out of these approaches, only three are associated with the mechanisms implemented in this project: Workload Control, just in time and theory of constrains.

The main purpose of Workload Control is to reduce the work in process, avoiding the overload of the production system. The second one, just in time, associated with Generic Kanban System, targets cost reduction. Finally, the Theory of Constrains, related to the Drum-Buffer-Rope mechanism, finds the critical work centre (bottleneck). This bottleneck constrains the performance of the whole production system.

\subsection{Production activity control mechanisms}

In PAC there are several mechanisms that can be implemented. The most referred ones are Base Stock System, Toyota Kanban System, Constant Work in Process, Paired-Cell Overlapping Loops of Cards with Authorization. However, as previously mentioned, the implemented mechanisms in this project are Workload Control, Generic Kanban System and Drum-Buffer-Rope, associated with a make-to-order production [3?].

\subsubsection{Workload Control}

Workload control is aimed at controlling the workload in the production system. Maintaining the workload of the system stable results in shorter and stabilized course times and, consequently, better delivery performances, when compared to systems without control [10].

Therefore, in this mechanism, jobs are not immediately released into the system. Instead, they wait in a pre-shop pool for authorization to be released, according to the workload levels. This authorization is only given whenever jobs follow certain Workload Control rules associated with the work centres of its course [1].

In addition, according to Wight (1970), WLC is based on an input/output control. Thereby, the input rate of work is balanced according to the output rate and jobs are only released when the previous ones are concluded. 


\subsubsection{Generic Kanbans System}

The Generic Kanban System is a mechanism based on the use of signals (denominated as Kanbans). The name of this mechanism is related to the usage of generic signals, which can be attributed to any job and not just to a specific one [5].

In each work centre, there are a number of signals available and specific to the different centres. Therefore, a job is only released into the production system when there is at least one Kanban available in every centre. Otherwise, it waits on the pre-shop pool. The Kanbans are allocated to jobs when they are released, and follow them until job processing is finished. Each time a job finishes its process on the work centre, Kanbans are dissociated and, thus, can be allocated to new jobs [11].

\subsubsection{Drum-Buffer-Rope}

The mechanism Drum-Buffer-Rope consists on identifying the constrainer work centre (bottleneck) of the production system to synchronize the production flow.

Metaphorically, once the drum (bottleneck) is identified, it defines the rhythm of the other work centres since it is the one with the highest load [4]. Thereby, the bottleneck is responsible for the performance of the whole production system [8]. Furthermore, rope controls raw material needs according to the bottleneck's capacity.

Similarly to the previous mechanisms, when jobs are not immediately released into the production system, they remain in the pre-shop pool until the authorization is given.

The bottleneck can vary over time. However, in this project, it will be constant.

\section{Experimental Plan and Key Performance Indicators}

To obtain simulation results, the different mechanisms are implemented in each machine selection criteria, creating nine independent models. Each model is analysed in ten scenarios. Those vary according to the mechanism: in WLC and DBR, the variable is the load norm imposed on the system $(6.2,7.3,8.6,10.1,11.9,14,16.5,19.4$, 22.8 and infinite - unrestrictive release of jobs); in GKS, it is the number of Kanbans $(7,8,9,10,12,14,17,20,23$ and infinite). In addition, it is established a warm-up period of 3000 hours, a replication length of 13000 hours and a number of replications of 100 .

Key Performance Indicators allow for a comparison among the mechanisms to conclude which one has the best performance:

- Shop Throughput Time (hours) is the average time between the release time of the job to the first work centre and its conclusion.

- Pool Time (hours) is the average waiting time of each job before entering the first work centre.

- Total Throughput Time (hours) is the average total time that a job remains in the system. It includes the STT and the Pool Time. 
- Lateness (hours) is the difference between the conclusion time of the job and its due date, on average.

- Standard Deviation of Lateness (Lateness St. Dev.) (hours) is a dispersion measure which represents the lateness deviation from the lateness average.

- Percentage Tardy is the proportion of late jobs in relation to all jobs.

\section{$4 \quad$ Results and Discussion}

Results are analysed according to two different perspectives: finding the best machine selection criteria for each mechanism (First Analysis), and finding the best mechanism for each machine selection criteria (Second Analysis).

The comparison is made through the results obtained from the KPIs on the different scenarios. Additionally, a Multivariate Analysis is performed to better understand how KPIs are related and their influence [13]. On the one hand, it is concluded, through the correlation matrix, that Lateness is strongly and positively correlated to Percentage Tardy and TTT. The same situation happens between Percentage Tardy and TTT. This indicates that three KPIs have similar behaviour in the same proportions. It can be also concluded that there are no negative correlations among the KPIs. On the other hand, through the analysis of the main components, an expected result is obtained: Lateness and Percentage Tardy are considered variables with extreme importance for the study. Furthermore, it is ascertained that the total system time (TTT) is more relevant than production system time (STT).

\subsection{First Analysis}

In this section, the results obtained for the three machine selection criteria (Random, Load Hours and Load Units) under the mechanisms are analysed and compared.

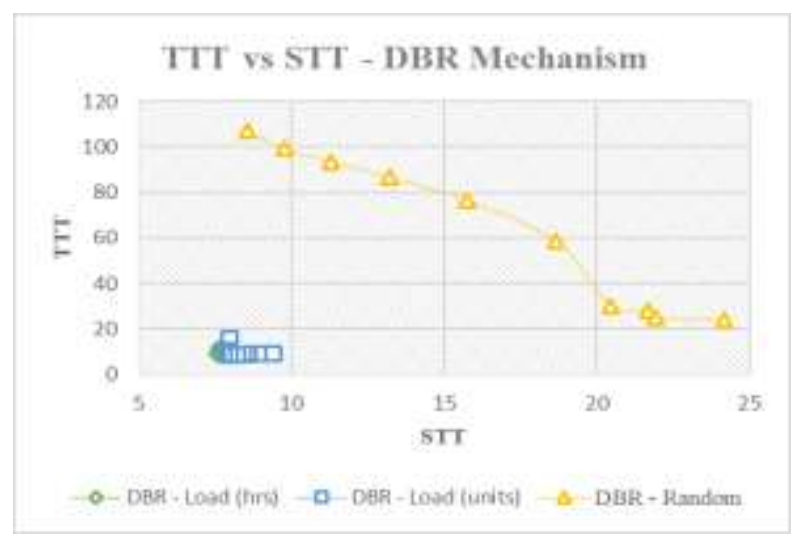

Fig. 2. Results for TTT vs. STT for the DBR mechanism. 


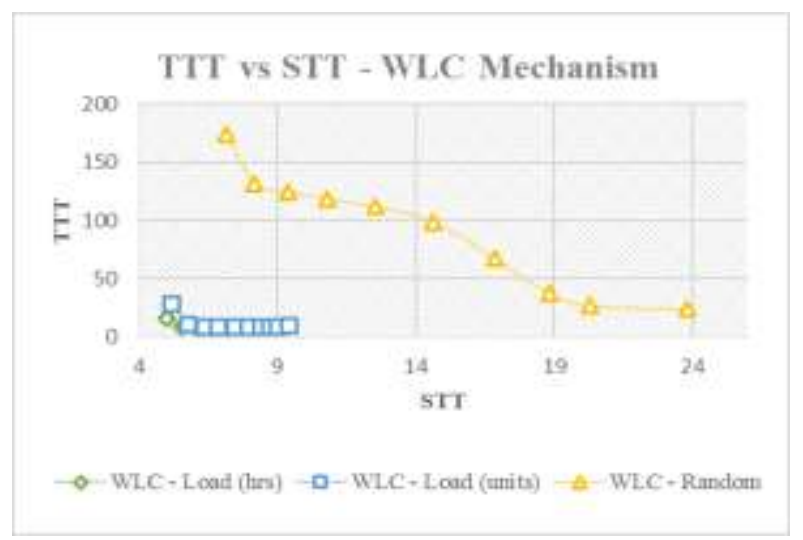

Fig. 3. Results for TTT vs. STT for the WLC mechanism.

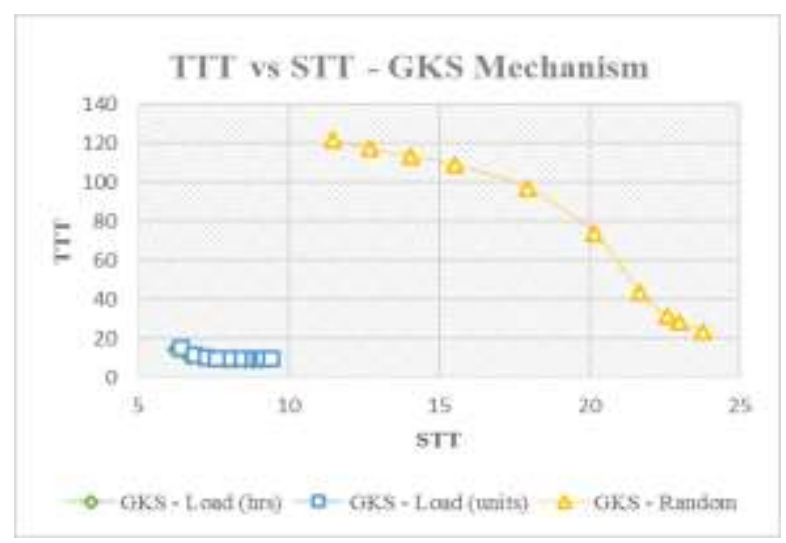

Fig. 4. Results for TTT vs. STT for the GKS mechanism.

As Figure 2 illustrates, Random is the machine selection criterion which differs in behaviour from the other criteria. It is the criterion with the highest values of STT and TTT, and has values of TTT above 100 hours for the smallest values of load norm/ Kanbans, and values of STT higher than 20 hours for the highest values of load norm/ Kanbans. This means that jobs under this criterion remain more time in the total and the production systems than the other criteria. It is also important to refer that, under the DBR mechanism, although TTT decreases drastically with the increment of the load norm/ Kanbans on the system on the Random criterion, it only reaches the highest values of the other two criteria in the highest values of load norm/ Kanbans. Likewise, the smallest value of STT of the Random criterion is higher than the highest values of the Load Hours and Units. 
Comparing Load Hours and Load Units to identify the one with the best performance on the three mechanisms, it can be observed that both curves present a similar behaviour. Nevertheless, Load Hours is the criterion with the smallest values of TTT and STT for the same scenarios, meaning that jobs remain less time in both total and production system.

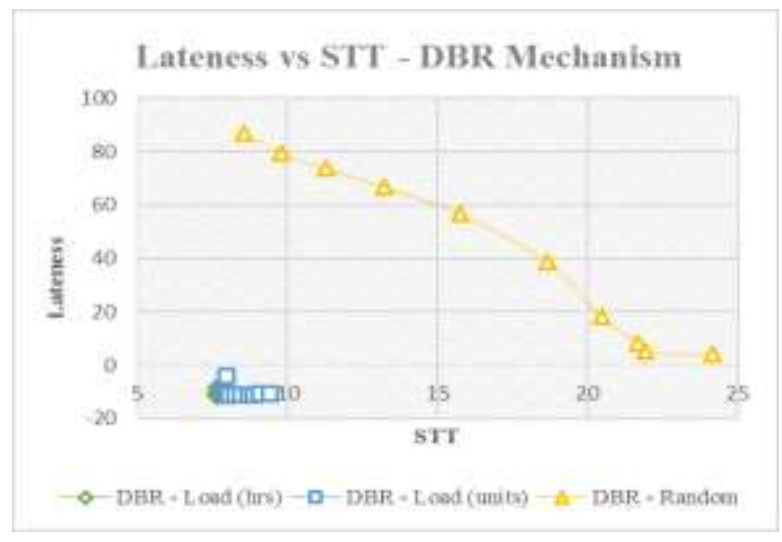

Fig. 5. Results for Lateness vs. STT in the DBR mechanism.

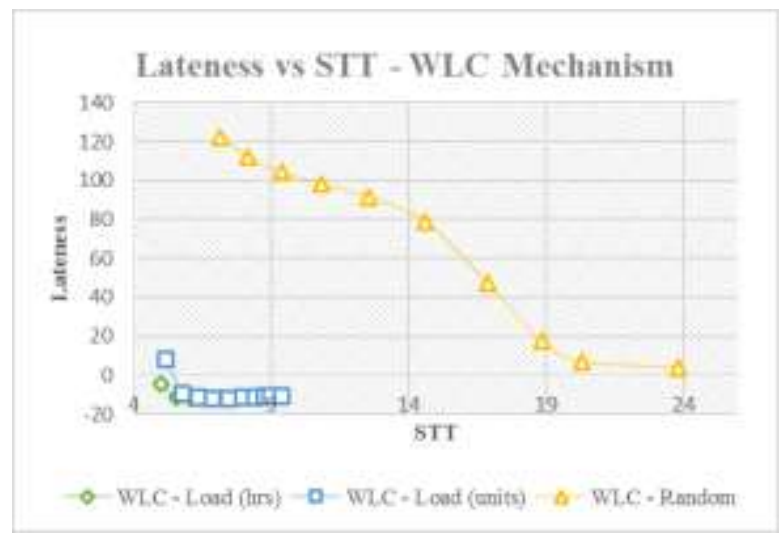

Fig. 6. Results for Lateness vs. STT in the WLC mechanism. 


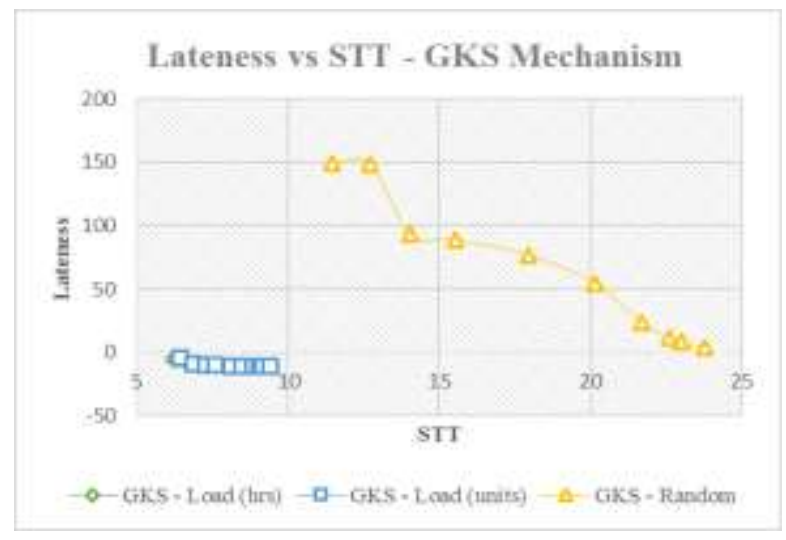

Fig. 7. Results for Lateness vs. STT in the GKS mechanism.

Regarding the average Lateness, on the one hand, Figure 3 shows that, once again, Random is the criterion with the highest values of this KPI, with all values positive for all the mechanisms, meaning that, on average, jobs are always late. However, the curves of this criterion decreases drastically with the increase of load norm/ Kanbans since there are no constraints in the production system when the load norm/ number of Kanbans available is infinite. Therefore, it can be concluded that, on average, even with the highest values of load norm/ Kanbans, jobs will never be on time under this criterion.

On the other hand, Load Hours is the criterion with the smallest values of average Lateness, and is the only one with just negative values, which means that, on average, jobs are always in advance. It can also be concluded that when values tend to infinite, values of average Lateness tend to stabilize because of the increasing production system fluidity.

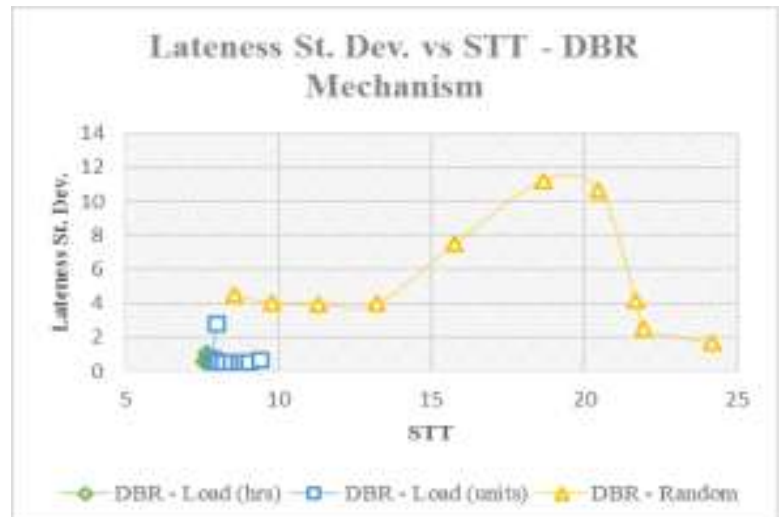

Fig. 8. Results for Lateness Standard Deviation vs. STT in the DBR mechanism. 


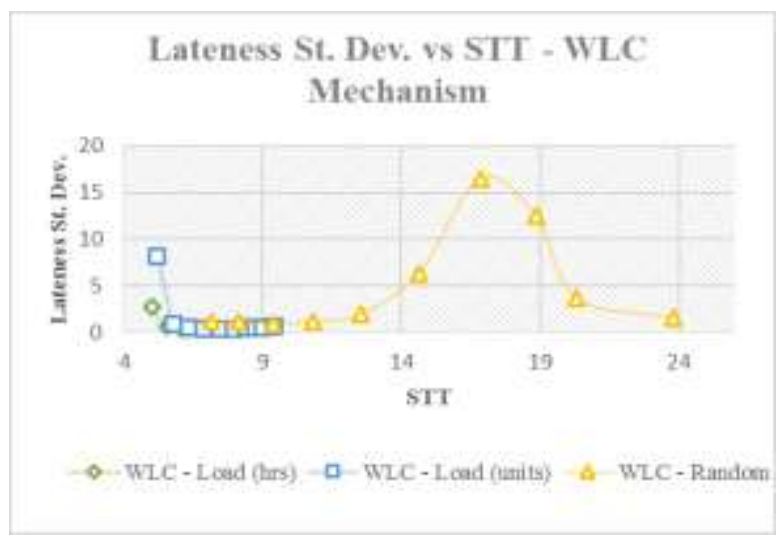

Fig. 9. Results for Lateness Standard Deviation vs. STT in the WLC mechanism.

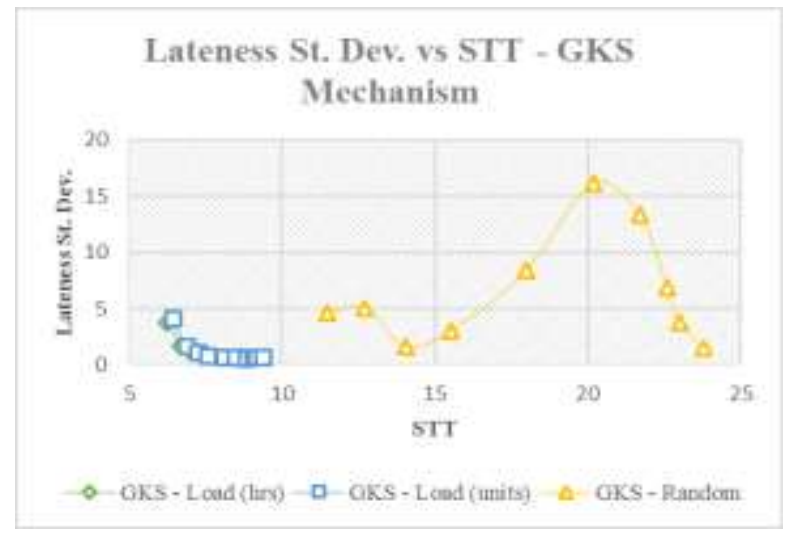

Fig. 10. Results for Lateness Standard Deviation vs. STT in the GKS mechanism.

Referring to Lateness Standard Deviation, as seen in Figure 4, Random is also the criterion with the highest values and variability of values, meaning that there is a large dispersion of Lateness values in relation to the average. It is important to notice that these values increase until the scenario where the load norm is 16.5 for WLC and 14 for DBR and GKS, decreasing afterwards, meaning that for higher values of load norm/ Kanbans, Lateness Standard Deviation is lower.

In addition, Load Units has a high value of Lateness Standard Deviation on the smallest value of load norm under the WLC and DBR mechanisms, meaning that, in this scenario, Lateness has a large dispersion of the values relatively to the average. This value is significantly different from the value observed on the same scenario for the other criterion. However, the remaining values are on the same range. It can be 
concluded that, for Load Hours and Load Units, values of Lateness Standard Deviation are low.

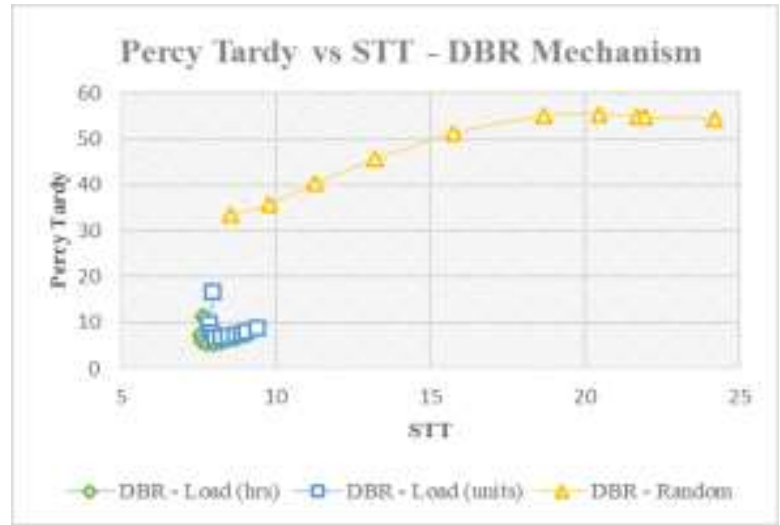

Fig. 11. Results for Percentage Tardy vs. STT in the DRB mechanism.

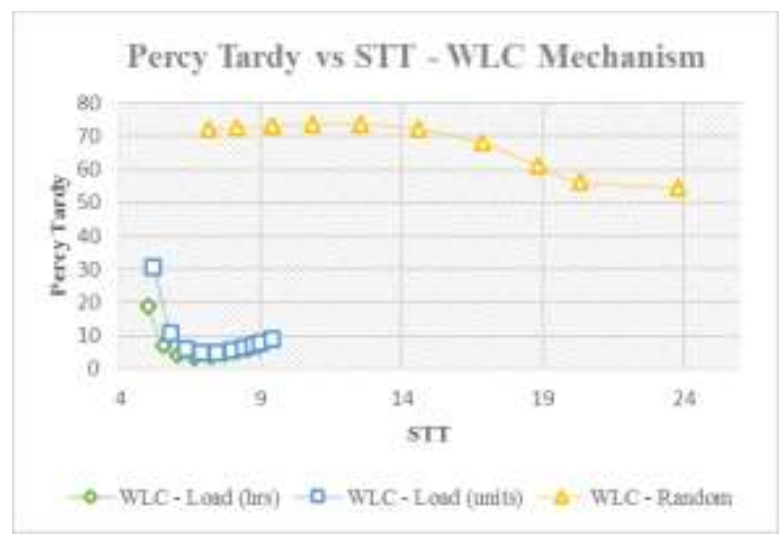

Fig. 12. Results for Percentage Tardy vs. STT in the WLC mechanism. 


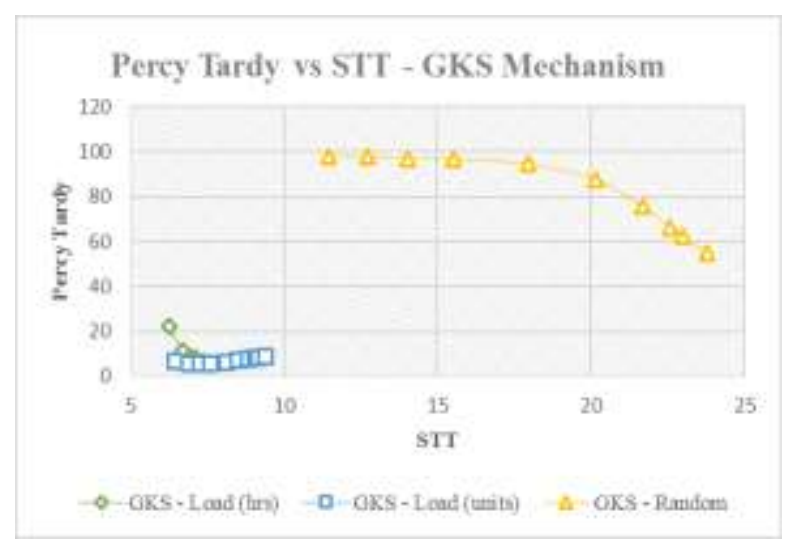

Fig. 13. Results for Percentage Tardy vs. STT in the GKS mechanism.

Lastly, Figure 5 shows that the values of Percentage Tardy for Random are always above $50 \%$, except for the DBR mechanism. Therefore, more than a half of the jobs are always late. Concerning the DBR mechanism, for this machine selection criterion, the registered values of Percentage Tardy increase with the increase of the load norm on the system, only decreasing in the last three scenarios, contrarily to the other mechanisms, and the highest value of this KPI founded is $55.46 \%$. However, for GKS, the highest value is $97.95 \%$.

Once again, Load Hours registers the smallest value of Percentage Tardy, and for DBR and WLC, Load Units has always higher values than Load Hours in all scenarios.

To conclude, while Random is the criterion with the worst performance for all the mechanisms, Load Hours is the one with the best results and performance in this case study.

\subsection{Second Analysis}

Firstly, it is important to refer that in the following analysis and for all graphics, curves converge to the same point. This occurs due to the fact that as the production time of jobs increases (STT), the total time in the system (TTT) tends to decrease and stabilize.

Theoretically, when the load norm and signals increase, jobs remain less time in the pre-shop pool because they do not have to wait for authorization to be released. Therefore, there is a decrease of the total time in the system and, in infinite, TTT equals STT (because of the dissolution of pool time).

Consequently, the mechanisms present all the same behaviour (convergence of curves) independently of the machine selection criteria implemented. 


\subsubsection{Random}

Subsequently, the results from the Random as a machine selection criterion will be explained.

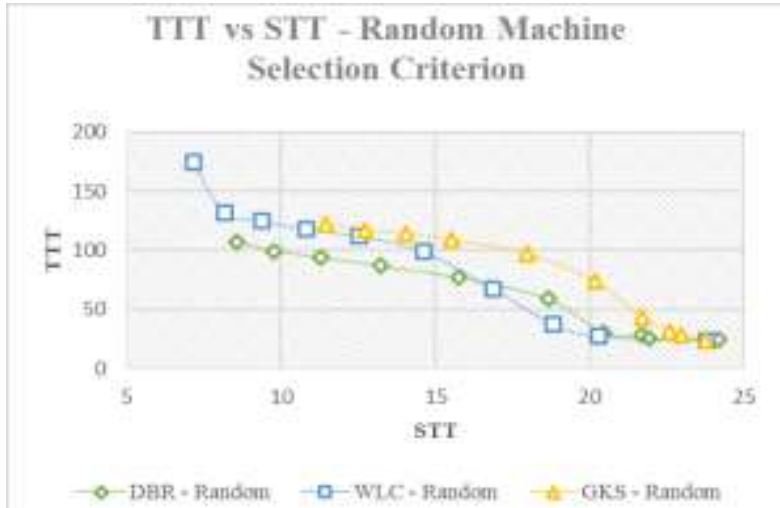

Fig. 14. Results for TTT vs STT with Random Machine Selection Criterion.

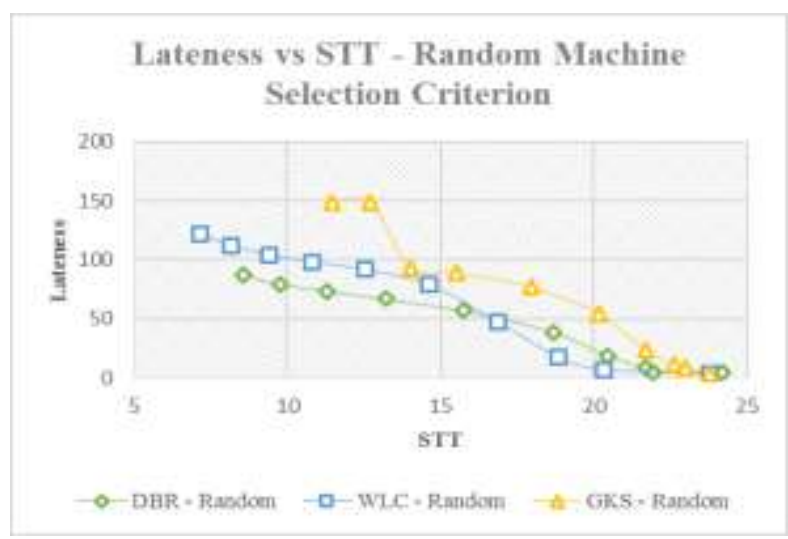

Fig. 15. Results for Lateness vs STT with Random Machine Selection Criterion. 


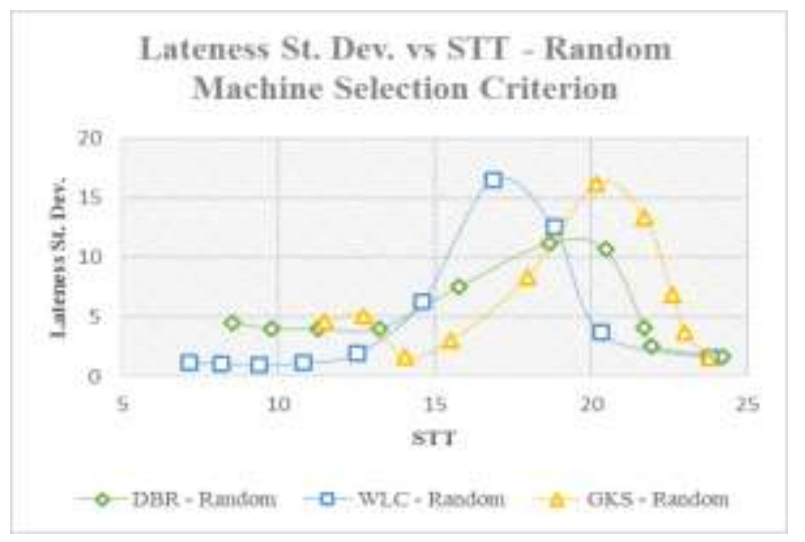

Fig. 16. Results for Lateness St. Dev. vs STT with Random Machine Selection Criterion.

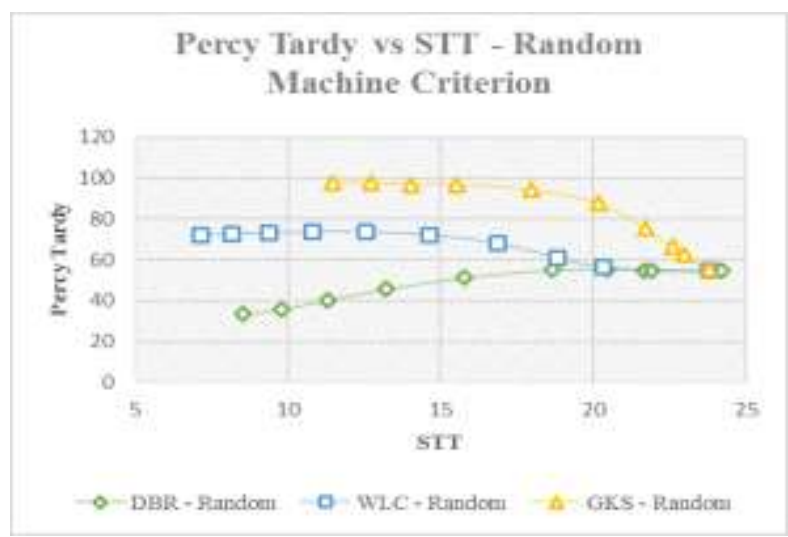

Fig. 17. Results for Percy Tardy vs STT with Random Machine Selection Criterion.

TTT vs. STT

As seen on top left of the Figure 6, all mechanisms behave similarly when the machine selection criterion is Random. However, through the TTT average for each mechanism, it can be concluded which one performs the best. In the DBR mechanism, the TTT average is 62.90 hours and the STT average is 16.56 hours; in the WLC mechanism, the TTT average is about 91.46 hours and the STT average is 14.25 hours; lastly, in the GKS mechanism, the TTT average is about 75.92 hours and the STT average is 18.29 hours. Therefore, the DBR is the mechanism under which products remain the least time in the total system. The WLC mechanism is where products stay the least time in the production system, but the most time in the total system. This indicates that products are waiting more time in the pre-shop pool under this mechanism in comparison to the others. 


\section{Lateness vs. STT}

Once again, the different curves that represent the different mechanisms behave analogously (top right of the Figure 6). As the load norm imposed to system increases, Lateness values decrease significantly. When the system has a higher load norm, it is possible to have jobs finished at the right time, registering minimums of Lateness of 3.80 hours (WLC), 3.78 hours (GKS) and 4.19 hours (DBR). On average, the mechanism that has the least number of late jobs is DBR (43.71 hours).

\section{Lateness Standard Deviation vs. STT}

The graphic on down left of the Figure 6 reveals how Lateness values vary across different load norm values. The highest values for all mechanisms are registered in the range of load norm $14-16.5$ hours. It can be seen that WLC and GKS present a higher dispersion of Lateness values from the average, while DBR presents constant values of Lateness Standard Deviation.

Percentage Tardy vs. STT

Regarding the Percentage Tardy results (down right of the Figure 6), it is possible to conclude, once again, that all mechanisms perform similarly. However, on average, DBR performs the best, since it presents the lowest value of Percentage Tardy $(48.09 \%)$. This result is expected, since the DBR mechanism has the lowest value of Lateness.

As the result of this brief analysis, DBR is considered the best one when the criterion is Random.

\subsubsection{Load Hours}

In this section, the results obtained for the three mechanisms on the Load Hours machine selection criterion are studied and compared.

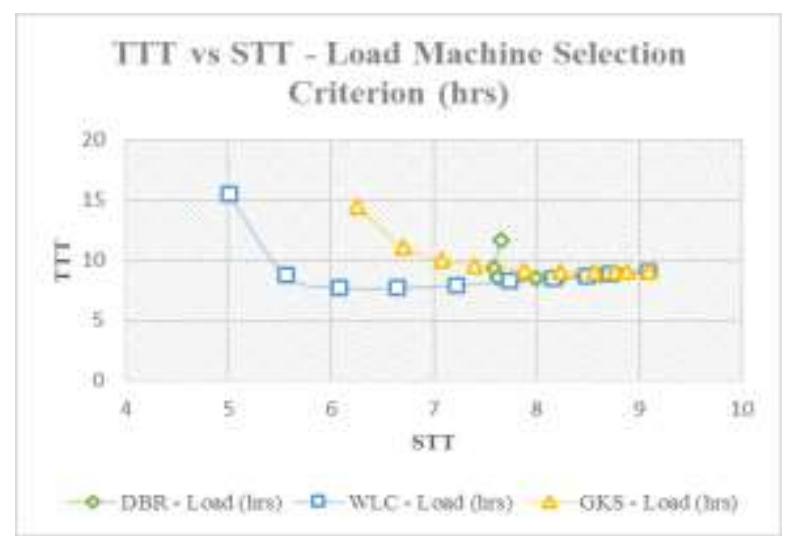

Fig. 18. Results for TTT vs STT with Load Hours Machine Selection Criterion. 


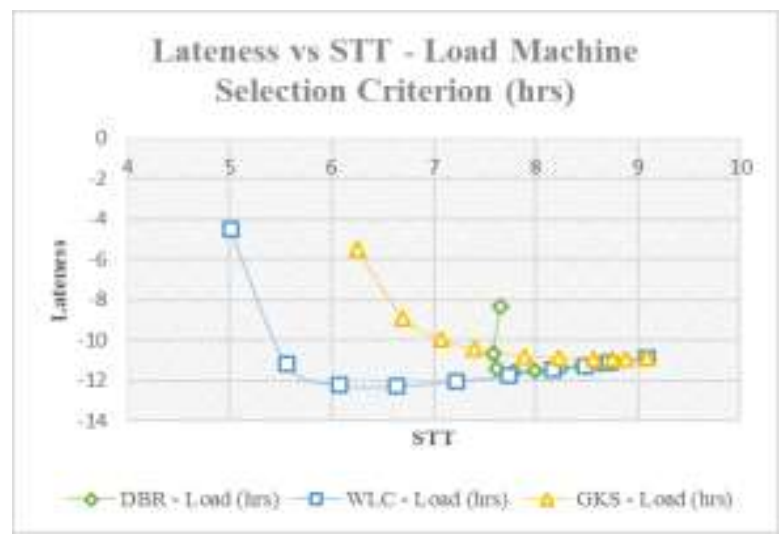

Fig. 19. Results for Lateness vs STT with Load Hours Machine Selection Criterion.

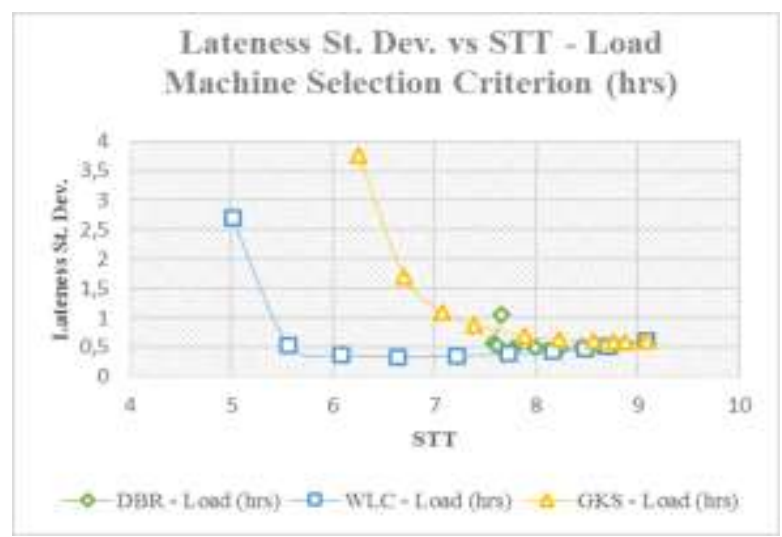

Fig. 20. Results for Lateness St. Dev. vs STT with Load Hours Machine Selection Criterion.

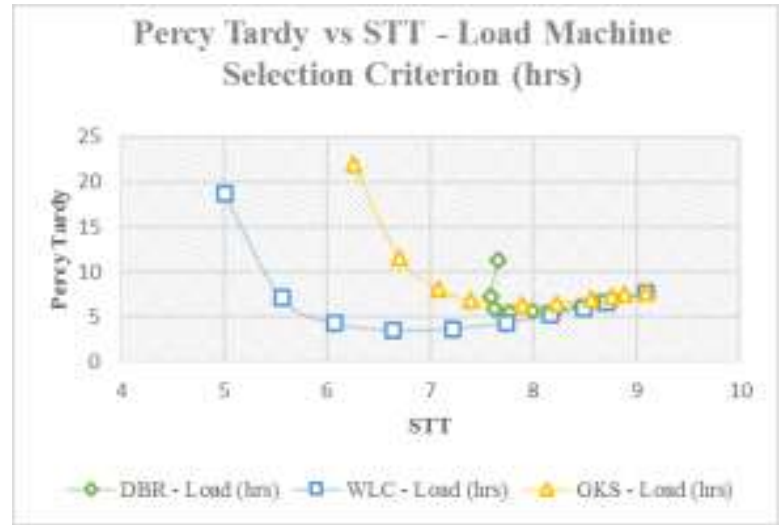

Fig. 21. Results for Percy Tardy vs STT with Load Hours Machine Selection Criterion. 
TTT vs. STT

Being the Load Hours the machine selection criterion, it is shown that the relation between TTT and STT is similar in the three mechanisms despite the range of the TTT values of each mechanism, as it can be seen on top left of the Figure 7. In all of them, the TTT average values are similar (TTT in DBR mechanism: 9.05 hours, TTT in WLC mechanism: 9.11 hours and TTT in GKS mechanism: 9.97 hours). Therefore, it can be concluded that products remain more time in the total system under the GKS mechanism, on average. The mechanism which registers the lowest value of TTT is WLC (7.76 hours). Despite that, it is under the DBR mechanisms that products remain, on average, the least time in the total system, although they remain the longest in the production system. This fact allows for the conclusion that the products remain less time in the pre-shop pool under the DBR than under the other mechanisms.

Lateness vs. STT

Regarding KPI Lateness (top right of the Figure 7), a strong correlation with KPI TTT $(\rho=1)$ is observed. Therefore, the minimum value of Lateness registered originates from the WLC mechanism (-12.30 hours). However, on average, the mechanism which results in a lower value of Lateness is DBR. All mechanisms present only negative values, indicating that products are in advance.

Lateness Standard Deviation vs. STT

In relation to Lateness Standard Deviation (graphic on down left of the Figure 7), the minimum value is registered under the WLC mechanism. Nevertheless, it can be visually observed that Lateness Standard Deviation moves further away from the average in the WLC and GKS mechanisms, leading to the conclusion that stable values of Lateness are obtained through the DBR mechanism.

\section{Percentage Tardy vs. STT}

Since the lowest Lateness values arise from DBR mechanism and the products remain the least time in the total and production systems, it is expectable that this mechanism has a lower percentage of late jobs. However, the WLC mechanism has, on average, a slightly lower percentage of late jobs $(6.68 \%)$ in comparison to the DBR (6.87\%). Regarding the GKS mechanism, it can be concluded that the percentage of late jobs is the highest in this mechanism (down right of the Figure 7).

Therefore, when the machine selection criterion is based on the Load Hours of the production system, the best performing mechanism is the DBR.

\subsubsection{Load Units}

Lastly, the results of Load Units machine selection criterion are presented. 


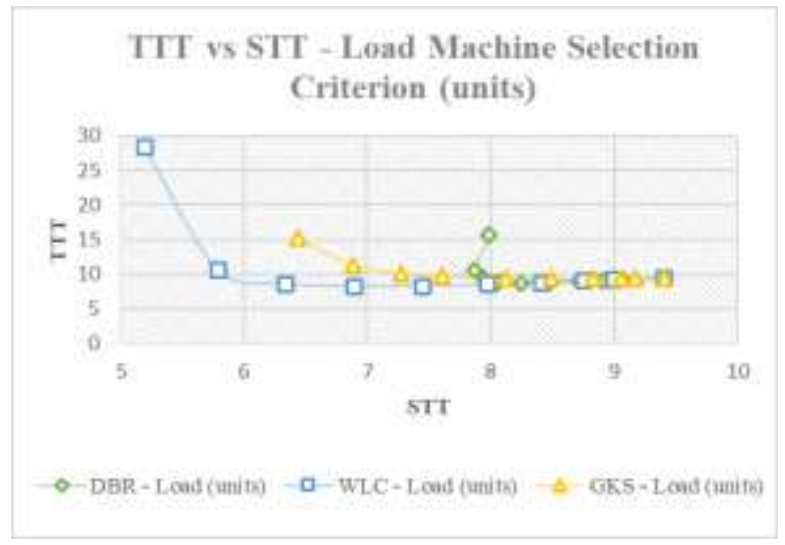

Fig. 22. Results for TTT vs STT in Load Units Machine Selection Criterion.

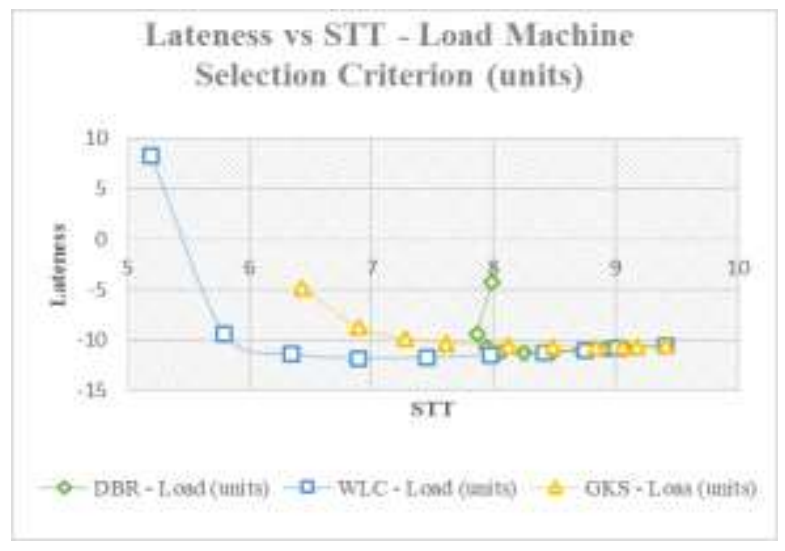

Fig. 23. Results for Lateness vs STT in Load Units Machine Selection Criterion.

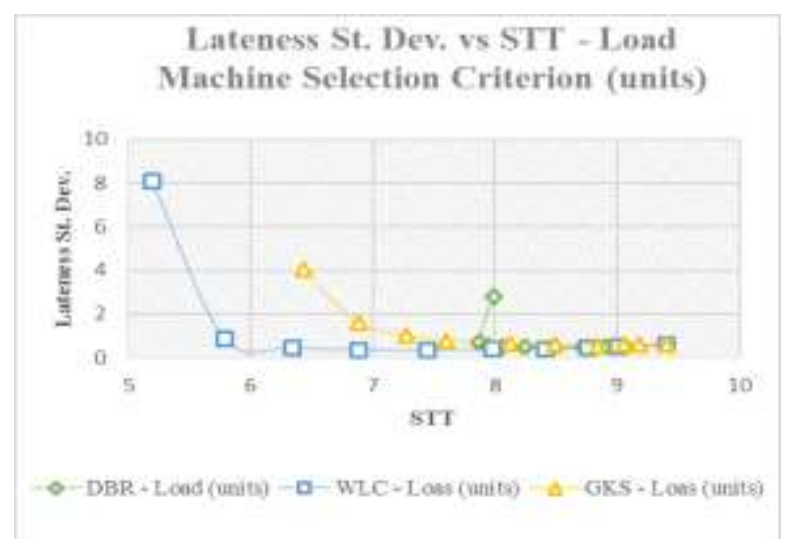

Fig. 24. Results for Lateness St. Dev. vs STT in Load Units Machine Selection Criterion. 


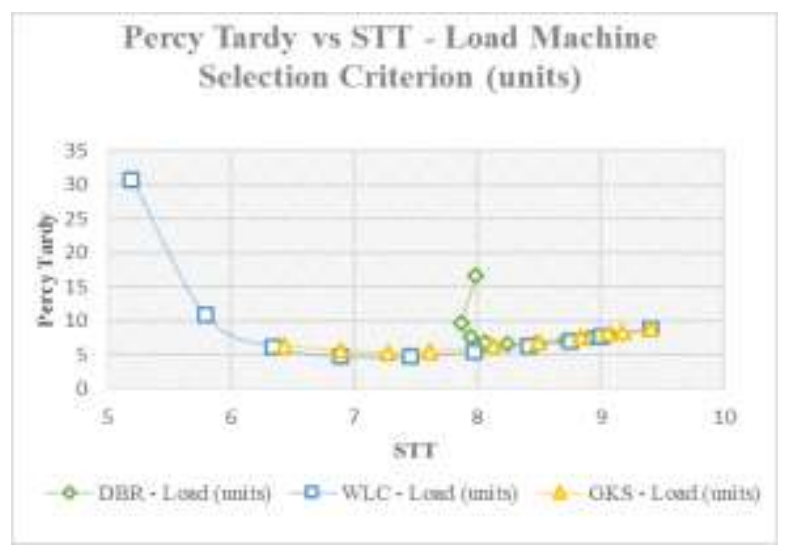

Fig. 25. Results for Percy Tardt vs STT in Load Units Machine Selection Criterion.

TTT vs. STT

Comparing the performances of the different mechanisms (graphic on top left of the Figure 8), the WLC mechanism has the lowest STT value (7.55 hours) and the highest value of TTT (10.88 hours), on average, implying that products remain more time in the pre-shop pool. However, this can be justified by the existence of an atypical value from the first scenario, where load norm is very low, which results in the high TTT value and explains why WLC has, simultaneously, the highest value of TTT and the lowest STT. In the other mechanisms, the same situation occurs: the averages of TTT are influenced by the first scenario. To evaluate the performances without that influence, the averages were recalculated excluding the first scenario, allowing for the conclusion that the WLC is the mechanism under which products remain the least time in the entire system (TTT: 9.95 hours) and also in the production system (STT: 7.78 hours). Though, DBR mechanism evidences a lower average value of TTT.

\section{Lateness vs. STT}

Through the analysis of the graphic on top right of the Figure 8, it can be verified that almost all jobs are concluded before their due date. It is through the WLC mechanism that the minimum Lateness value is obtained ( -11.81 hours).

Similarly to the previous situation, if the first observation of the WLC mechanism were to be omitted, this mechanism would have the lowest average value of Lateness. However, considering the general panorama, it is under the DBR mechanism that products are the most advanced.

Comparing the curves TTT vs. STT and Lateness vs. STT, a similarity can be observed. Using the Spearman Correlation between TTT and Lateness, it can be concluded that in the DBR mechanism $\rho=1$, in the GKS mechanism $\rho=1$ and in the WLC mechanism $\rho=1$. These correlations indicate the existence of a linear and positive relation between these variables, since TTT is the total time in the entire system and Lateness represents the subtraction of the instant when a job is completed from its due date. 
Lateness Standard Deviation vs. STT

In all mechanisms (down left of the Figure 8), the first value presents the highest Lateness variation and the remaining values mostly have a Lateness Standard Deviation under one hour. That result indicates that, except for the first scenario, there is a little dispersion of Lateness values regarding the average.

Percentage Tardy vs. STT

Although the WLC mechanism presents some values lower than other mechanisms, the GKS mechanism is the one with the lowest average percentage of late jobs, as it can be seen on down right of the Figure 8. This does not match the previous conclusions regarding Lateness, since it was previously observed that the DBR mechanism had the lowest value of late jobs. To analyse this situation, the Spearman correlation between Percentage Tardy and Lateness is used: DBR $-\rho=0.98$; GKS $-\rho=-0,61$. In the DBR mechanism, as Lateness increases or decreases, the percentage of late jobs change in the corresponding proportion. The opposite situation occurs in the GKS mechanism: the increase of the Lateness of the jobs corresponds to a decrease in Percentage Tardy, and vice-versa. Despite having a lower Percentage Tardy, the GKS presents higher job lateness when compared to the DBR.

Considering the Load Units as a machine selection criterion, it is concluded that the DBR has the best performance. However, WLC would be better in all KPIs if the first scenario was excluded.

\section{Conclusion}

After all the analysis performed, it can be concluded that the DBR is the mechanism with the best performance in almost all the studied scenarios. Even though it is the mechanism which has the highest values of STT (since jobs do not have to wait for authorization of all stages, meaning they remain less time in the pool and are released into the production system more easily than in the other mechanisms), it has the smallest values of both TTT (which means that jobs remain less time in the total system and in the pre-shop pool) and Lateness. In addition, even though it is not the best mechanism in terms of Percentage Tardy, the DBR still presents quite satisfactory values.

The previous conclusion can be related to the fact that this mechanism has a fixed bottleneck. In this project, stage 1 is considered the permanent bottleneck, since it is the stage in which products queue up to enter the production system. After that, the products flow without influence of any mechanism.

However, it is also important to note that the scenario with the lowest load norm is compromising the performance of WLC, since it has better performance in the other scenarios.

Regarding the machine selection criteria, Load Hours performs the best since it has the smallest values of almost all KPIs. Contrarily, Random is the criterion with the 
worst performance. This result is expected because Load Hours is the only criterion that has the workload of the machines in consideration. Therefore, in similar contexts companies should consider the implementation of Load Hours instead of the other criteria.

Suggestions for further developments include the implementation of DBR with a non-constant bottleneck, verifying which stage has the highest load throughout the production time to identify the bottleneck in real time. In addition, using the Optquest study in Simio can be important to obtain the optimum number of Kanbans and load norm.

\section{References}

1. Akillioglu, H., Dias-Ferreira, J., \& Onori, M. (2016). Characterization of continuous precise workload control and analysis of idleness penalty. Computers \& Industrial Engineering, 102, 351-358, DOI:10.1016/j.cie.2016.05.026

2. Burbidge, J. L. (1990). Production control: a universal conceptual framework. Production Planning \& Control, 1(1), 3-16

3. Carmo-Silva, S. (2010). Textos de Gestão da Produção, v01_10. Publicação Interna Departamento de Produção e Sistemas, Universidade do Minho, Braga.

4. Chakravorty, S., \& Atwater, J. (2005). The impact of free goods on the performance of drum-buffer-rope scheduling systems. International Journal of Production Economics, 95, 347-357. DOI:10.1016/j.ijpe.2004.01.001

5. Chang, T., \& Yih, Y. (1994). Generic kanban systems for dynamic environments. International Journal of Production Research, 32, 889-902. DOI:10.1080/00207549408956977

6. Chen, B., \& Matis, T. (2013). A flexible dispatching rule for minimizing tardiness in job shop scheduling. International Journal of Production Economics, 141, 360-365. DOI:10.1016/j.ijpe.2012.08.019

7. Fernandes, N. O., Thürer, M., Silva, C., \& Carmo-Silva, S. (2016). Improving workload control order release: Incorporating a starvation avoidance trigger into continuous release. International Journal of Production Economics.

8. Georgiadis, P., \& Politou, A. (2013). Dynamic Drum-Buffer-Rope approach for production planning and control in capacitated flow-shop manufacturing systems. Computers \& Industrial Engineering, 65, 689-703. DOI:10.1016/j.cie.2013.04.013

9. Hermann, M. P. (2016, January). Design principles for industrie 4.0 scenarios. In System Sciences (HICSS), 2016 49th Hawaii International Conference on (pp. 3928-3937). IEEE

10. Kingsman, B., \& Hendry, L. (2002). The relative contributions of input and output controls on the performance of a workload control system in make-to-order companies. Production Planning \& Control, 579-590. DOI:10.1080/0953728021000026285

11. Lage Junior, M., \& Godinho Filho, M. (2010). Variations of the kanban system: Literature review and classification. International Journal of Production Economics, 125, 13-21. DOI:10.1016/j.ijpe.2010.01.009

12. Posada, J., Toro, C., Barandiaran, I., Oyarzun, D., Stricker, D., de Amicis, R., . . . Vallarino, I. (2015). Visual Computing as a Key Enabling Technology for Industrie 4.0 and Industrial Internet. IEEE COMPUTER GRAPHICS AND APPLICATIONS, 35, 26-40.

13. Reis, E. (1997). Estatística Multivariada e Aplicações. Lisboa: Edições Sílabo, Lda. 
14. Stevenson, M., Hendry, L., \& Kingsman, B. (2005). A review of production planning and control: the applicability of key concepts to the make-to-order industry. International Journal of Production Research, 43, 869-898. doi:10.1080/0020754042000298520

15. Stock, T., \& Seliger, G. (2016). Opportunities of Sustainable Manufacturing in Industry 4.0. Procedia CIRP, 40, 536-541. DOI:10.1016/j.procir.2016.01.129 